\section{The OnControl bone marrow biopsy technique is superior to the standard manual technique for hematologists-in-training: a prospective, randomized comparison}

\author{
Louis Juden Reed, ${ }^{1}$ Radha Raghupathy, ${ }^{2}$ \\ Marianna Strakhan, ${ }^{1}$ \\ Thomas E. Philbeck, ${ }^{3}$ Mimi Y. Kim, ${ }^{4}$ \\ Ramakrishna Battini, ${ }^{1,2}$ \\ Zulfiqar Hussain, 1,2 Shaad Abdullah,,2 \\ Sarah Schweber, ${ }^{1,2}$ Kamalesh Bala, ${ }^{1,2}$ \\ Thomas Pacello ${ }^{1}$ \\ ${ }^{1}$ Albert Einstein College of Medicine, \\ Jacobi Medical Center, ${ }^{2}$ Division of \\ Hematology and Department of \\ Oncology, Montefiore Medical Center, \\ Bronx, NY, ${ }^{3}$ Vidacare Corporation \\ Shavano Park, TX, ${ }^{4}$ Albert Einstein \\ College of Medicine, Bronx, NY, USA
}

\section{Abstract}

The purpose of this study was to compare a novel bone marrow device with the standard marrow needle in a prospective, randomized study in a teaching hospital employing hematologists-in-training. The new device, the OnControl Bone Marrow (OBM) Biopsy System, utilizes a battery-powered drill to insert the needle. Fifty-four bone marrows (27 standard and $27 \mathrm{OBM}$ ) were performed by 11 fellows under the observation and supervision of 3 attending hematologists and 1 research technologist. The primary endpoint of the study, the mean length of the marrow biopsy specimens, a surrogate for marrow quality, was determined by a pathologist in a blinded manner. The mean length of the marrow biopsy specimens was significantly longer (56\%) for the OBM group $(15.3 \mathrm{~mm})$ than for the standard bone marrow (SBM) group $(9.8 \mathrm{~mm})$, $\mathrm{P}<0.003$. An objectively determined secondary endpoint; mean procedure time, skin-to-skin; also favored the OBM group (175 s) versus the SBM group (292 s), $\mathrm{P}<0.007$. Several subjective secondary endpoints also favored the OBM group. Only minor adverse events were encountered in the OBM and SBM study groups. It was concluded that bone marrow procedures (BMPs) performed by hematologists-in-training were significantly faster and superior in quality when performed with the OBM compared to the SBM. These data suggest that the OBM may be considered a new standard of care for adult hematology patients. OBM also appears to be a superior method for training hematology fellows.

\section{Introduction}

The human bone marrow is often evaluated in patients with various hematological disorders, including anemia, thrombocytopenia, pancytopenia, leukemia and other hematological malignancies. ${ }^{1}$ Multiple bone marrow procedures (BMP) are often required in patients with hematological malignancies to guide their treatment. The instrument customarily employed, the Jamshidi needle, is designed to yield both an aspirate and a marrow biopsy. The BMP has changed very little in the last 40 years and involves a manual rotary insertion of the Jamshidi needle into the marrow cavity of the posterior aspect of the iliac bone. Although local anesthesia for the skin, subcutaneous tissues and periosteum is universally administered, the BMP is regarded by patients and physicians alike as a painful and uncomfortable procedure..$^{2-4}$ In addition, suboptimal specimens including dilute aspirates and small core biopsies are often obtained, limiting the diagnostic potential of the procedure.

The conventional manual rotary technique causes excessive periosteal stimulation contributing to the pain. In addition, especially in heavy patients, the biopsy length is often suboptimal due to limitation of depth that can be reached in the bone by manual pressure. A new FDA-approved device for performing bone marrows, the 0nControl Bone Marrow Biopsy System (OBM) was recently introduced by the Vidacare Corporation (Shavano Park, TX, USA). The OBM utilizes a battery-powered drill to insert the marrow needle into the iliac bone of adult hematology patients. Initial clinical studies utilizing the OBM system indicated that it was faster and easier to use for bone marrow aspirations than the traditional method. ${ }^{5}$ A few prospective studies comparing the OBM with the standard bone marrow procedure (SBM) have been carried out to date..$^{6-8}$ While the duration of the procedure has been consistently shorter, and the core samples larger for patients undergoing OBM in these reports, no studies have been carried out in teaching hospitals to determine whether or not the OBM system will be more readily mastered by hematologists-in-training. Instruction of hematology fellows in the BMP technique has never been standardized, varies greatly between different fellowship programs and seldom receives high priority. As a result, fellows completing training are sometimes not well versed in the performance of the BMP and tend to avoid it in their post-fellowship careers. We conducted a prospective, randomized study to compare the OBM procedure with the SBM procedure in adults. The length of marrow biopsy specimens (a surrogate for marrow quality), aspirate quality and spicule content and procedure time were assessed objectively
Correspondence: Louis J. Reed,

Jacobi Medical Center, Division of Hematology/ Oncology, 1400 Pelham Parkway South Suite 3N20 Bronx, NY 10461 USA.

Tel. +1.718.918.4581 - Fax: +1.718.918.5518.

E-mail: louis.reed@nbhn.net

Key words: bone marrow biopsy, core biopsy, trephine biopsy, powered biopsy, hematologistsin-training.

Acknowledgement: the authors would like to express appreciation for the others that contributed to the success of this study. These include: Anna Melissa Murillo MD, Amy Sharma MD, Venu Thirukonda MD, Murali Janakiram MD, and Carolina Schinke MD

Contributions: LJR, study conception and design, data analysis and interpretation, manuscript writing; RR, study conception, data analysis and interpretation, text reviewing; MS, study conception and design, data analysis and interpretation, text reviewing; TEP, study conception and design, data analysis and interpretation, manuscript writing; MYK, data analysis and interpretation, text reviewing; RB, ZH, SA, SS, KB, study conception, data collection and interpretation, text reviewing; TP, study conception, data analysis and interpretation, text reviewing.

Conflict of interest: LJR, RR, MS, MYK, and TP (or their organizations) have accepted research grant funds from Vidacare Corporation, the sponsor of the study and manufacturer of one of the study devices; TEP is an employee of Vidacare Corporation.

Received for publication: 23 August 2011. Accepted for publication: 12 October 2011.

This work is licensed under a Creative Commons Attribution NonCommercial 3.0 License (CC BYNC 3.0).

CCopyright L.J. Reed et al., 2011

Licensee PAGEPress, Italy

Hematology Reports 2011; 3:e21

doi:10.4081/hr.2011.e21

by the attending hematologist and pathologist. The patient, fellow and attending also completed questionnaires grading the pain, procedure difficulty, specimen quality and patient acceptability .

\section{Materials and Methods}

The study protocol was approved by the Biomedical Research Alliance of New York Institutional Review Board. Two kits were used, the OBM and SBM. The OBM system consists of a battery-powered driver and a biopsy needle set. The driver resembles a small 
hand-held drill, and powers a single lumen needle into the medullary cavity of the adult iliac bone. The needle set consists of two parts: an outer cannula, 11 gauge by 4 or 6 inches (102 or $152 \mathrm{~mm}$ ) long; and a bevel-tip inner stylet- used to penetrate the cortex. The SBM device used in the study was typically a Jamshidi bone marrow biopsy needle (11 gauge by 4 or 6 inches), which has a two-piece T-handle design, a trocar-tapered stylet point and a triple-crown cannula tip. All fellows satisfactorily completed at least one SBM and $\mathrm{OBM}$ procedure under the supervision of an attending hematologist, before they were deemed certified to start enrolling to the study. Each fellow was initially randomized to perform either the SBM or the OBM. After the initial randomization the fellow then alternated between OBM and SBM procedures in a sequential fashion. Randomization was performed for the fellows rather than the patients to minimize differences between individual fellows as to previous experience and variable aptitude.

After giving informed consent, adult patients requiring bone marrow sampling procedures underwent either a SBM or an OBM. Bone marrow aspiration and core biopsy were obtained utilizing a one needle/one puncture approach. The fellows were observed and supervised throughout the procedure by an attending hematologist or research technologist.

The planned sample size was 102 patients, 51 by each technique. The primary endpoint of the study was the mean length in millimeters of the bone marrow specimens yielded by the two techniques. The measured length of the marrow biopsy specimen is a generally accepted surrogate for the quality of the marrow biopsy. It was chosen as the primary endpoint because that measurement was made in the pathology department by a person who had no knowledge of whether the specimen was OBM or SBM and who was not involved in the study in any way.

Secondary endpoints included the skin to skin procedure time in seconds as well as other endpoints derived from the questionnaires. The questionnaires were completed by the patients, fellows and the attending hematologist/research technologist immediately after the procedure. All questions were answered on a 0 to 10 numerical scale. The patient questionnaire included questions regarding the level of pain experienced, the patient-perceived ease/difficulty of the procedure and the degree of patient willingness to have a repeat BMP if medically recommended. The questionnaires completed by the fellows included questions on the patient's level of pain, the ease/difficulty of the procedure and the perceived quality of the bone marrow aspirate and biopsy obtained. The questionnaires completed by the attending hematologist or by the research hematology technologist (who observed the procedure) included questions regarding the patient's apparent level of pain, the ease/difficulty of the procedure and the perceived quality of the bone marrow aspirate and biopsy obtained. Complications or adverse events were recorded during the procedure and at patient follow-up evaluation. Outpatients were called 1 or 2 days after the BMP and their responses recorded. Inpatients were visited 1 or 2 days after the BMP.

Statistical testing was conducted using SAS Version 9 for Windows (SAS Institute, Cary, NC). Continuous parameters were summarized and compared between groups using a 2 sample $t$-test. Categorical parameters were summarized as proportions and compared using Fisher's Exact test. Because most fellows used OBM and SBM multiple times, linear mixed effects models were fit to the data to evaluate differences between the OnControl and Standard methods and adjust for the potential correlation in repeated measurements from the same fellow. A priori significance level was set at 0.05 . An interim analysis was planned after the first 51 patients were accrued to determine if significant endpoints had been reached to permit early termination of the study.

\section{Results}

Two hospitals in the Bronx, NY participated in the study, Jacobi Medical Center and Montefiore Medical Center. A total of 54 BMPs (27 SBM and 27 OBM) were performed by 11 hematology fellows under the observation of 3 attending hematologists and 1 research technologist. The mean age of the 54 patients was $58.9( \pm 15.1)$ years and $61.1 \%$ were male. The mean height and weight were $168.1( \pm 11.0)$ cm and $77.9( \pm 19.3) \mathrm{kg}$, respectively. For these demographic parameters, there was statistical homogeneity between the two groups (Table 1). Of the 54 patients in the study (Table 2), 11 (20.4\%) had myeloma, the most frequentlyoccurring diagnosis. There was no significant difference in the frequency of diagnoses between the two groups $(\mathrm{P}=0.563)$.

The primary study endpoint, the mean marrow biopsy length was significantly longer in

Table 1. Patient demographics.

\begin{tabular}{lccc} 
Demographic & SBM & OBM & P \\
Number of male/female & $16 / 11$ & $16 / 11$ & 0.609 \\
Mean age (years \pm standard deviation) & $60.7 \pm 16.3$ & $57.2 \pm 14.0$ & 0.399 \\
\hline Mean height (cm \pm standard deviation) & $167.4 \pm 10.3$ & $168.0 \pm 12.1$ & 0.842 \\
Mean weight (kg \pm standard deviation) & $76.3 \pm 17.0$ & $78.8 \pm 22.2$ & 0.634 \\
\hline Body Mass Index & $27.2 \pm 5.6$ & $27.7 \pm 6.1$ & 0.755 \\
Race/Ethnicity: numbers of: & & & 0.730 \\
Black & 14 & 10 & \\
Hispanic & 9 & 12 & \\
Asian & 2 & 3 & \\
White & 2 & 2 & \\
\hline
\end{tabular}

Table 2. Patient diagnoses.

\begin{tabular}{lcc} 
Diagnosis & SBM & OBM \\
Myeloma & 7 & 4 \\
Pancytopenia & 3 & 5 \\
\hline Lymphoma, non-Hodgkin's & 3 & 3 \\
Anemia & 2 & 5 \\
\hline Myeloproliferative disorder & 3 & 2 \\
Monoclonal gammopathy of undetermined significance & 2 & 2 \\
\hline Myelodysplastic syndrome & 1 & 3 \\
Thrombocytopenia & 2 & 1 \\
\hline Hodgkins lymphoma & 1 & 1 \\
Acute myeloid leukemia & 1 & 0 \\
\hline IgM paraprotein & 1 & 0 \\
Metastatic carcinoma & 1 & 0 \\
\hline Eosinophilia & 0 & 1
\end{tabular}


the OBM group $(15.3 \mathrm{~mm})$ than in the SBM group $(9.8 \mathrm{~mm}), \mathrm{P}<0.003$ (Table 3$)$. The mean procedure time, a secondary endpoint, was significantly shorter in the OBM group (175 s) compared to the SBM group (292 s), $\mathrm{P}<0.007$.

Other secondary endpoints which significantly favored the OBM group included the mean pain scores recorded by the fellows $(\mathrm{P}<0.002)$ and by the attendings $(\mathrm{P}<0.008)$. In regard to the mean pain scores reported by the patients in the 2 study groups, a lower score was tabulated in the OBM group (4.7) than in the SBM group (5.9), but the difference was not significant $(\mathrm{P}=0.11)$. However, patients indicated a greater willingness to have a repeat OBM (1.0) than a repeat SBM (2.9), $\mathrm{P}<0.03$. Both the fellows (2.6 vs. 5.0, $\mathrm{P}<0.002$ ) and attendings ( 3.0 vs $5.1, \mathrm{P}<0.006)$ perceived the OBM to be easier to perform than the SBM. The attendings, but not the fellows, reported superior biopsy specimens in the OBM vs. the SBM group. The superiority of the OBM specimens was verified by objective blinded measurements in the pathology department. However, the fellows and the attendings scored the quality of the marrow aspirates as about equal. There were a greater number (proportion) of aspirates scored 0 (dry tap) in the OBM group (7/25\%) than in the SBM group $(4 / 15.4 \%)$, but the difference was not significant $(\mathrm{P}=0.505)$.

There were no serious adverse events in either the OBM or the SBM study groups. One patient withdrew consent after an OBM was begun. The needle had penetrated the cortex of the iliac bone, but the needle could not be detached from the driver in order to proceed with the aspiration and biopsy. That needle was withdrawn from the ilium. The BMP was subsequently performed without incident using a standard marrow needle. The event was classified as a device-related complication. Later, the manufacturer modified the OBM needle attachment. After that, no further incidents of that type were reported.

A minor adverse event occurred in a 56 year old man hospitalized for gastrointestinal bleeding, cirrhosis with portal hypertension, thrombocytopenia and coagulation abnormalities. He was being treated with red cell transfusions and fresh frozen plasma. Five days after an OBM, there was a spontaneous local drainage of a soft tissue hematoma from the posterior iliac marrow biopsy site. This responded to local and systemic therapy; the patient improved and was subsequently discharged. Another minor adverse event occurred in a 50 year-old female undergoing an outpatient SBM, which was successfully completed. Immediately after the procedure, the patient complained of numbness and weakness in the right lower extremity and was unable to stand. The patient was transferred via wheel chair to the emergency department,

Table 3. Study results: means \pm standard deviation.

\begin{tabular}{|c|c|c|c|}
\hline Variable & OBM & SBM & $\mathbf{P}$ \\
\hline \multicolumn{4}{|l|}{ Objective } \\
\hline \multicolumn{4}{|l|}{ Device efficacy } \\
\hline $\begin{array}{l}\text { Biopsy specimen length (mm) } \\
\text { (primary endpoint) }\end{array}$ & $15.3 \pm 7.3$ & $9.8 \pm 6.7$ & $0.003 *$ \\
\hline Procedure time (seconds) & $174.6 \pm 105.1$ & $292.1 \pm 210.0$ & $0.007 *$ \\
\hline \multicolumn{4}{|l|}{ Subjective } \\
\hline \multicolumn{4}{|l|}{ Pain Scores 0-10 } \\
\hline Perceived by patient & $4.7 \pm 2.8$ & $5.9 \pm 2.8$ & 0.11 \\
\hline Reported by fellow & $3.2 \pm 2.2$ & $4.9 \pm 2.7$ & $0.002 *$ \\
\hline Reported by attending & $2.9 \pm 1.7$ & $4.5 \pm 2.4$ & $0.008 *$ \\
\hline $\begin{array}{l}\text { Willingness by patient to repeat BMP } \\
\text { Procedure ease/difficulty }(0-10)\end{array}$ & $1.0 \pm 2.2$ & $2.9 \pm 3.5$ & $0.03^{*}$ \\
\hline Reported by patient & $1.1 \pm 1.7$ & $2.2 \pm 3.3$ & 0.11 \\
\hline Reported by fellow & $2.6 \pm 2.6$ & $5.0 \pm 2.8$ & $0.002 *$ \\
\hline Reported by attending & $3.0 \pm 2.6$ & $5.1 \pm 3.4$ & $0.006^{*}$ \\
\hline \multicolumn{4}{|l|}{ Perception of specimen adequacy $(0-10)$} \\
\hline Aspirate- fellow & $5.0 \pm 3.3$ & $5.4 \pm 2.7$ & 0.59 \\
\hline Aspirate- attending & $4.9 \pm 3.6$ & $5.6 \pm 3.1$ & 0.47 \\
\hline Core biopsy-fellow & $6.4 \pm 1.9$ & $5.1 \pm 3.1$ & 0.07 \\
\hline Core biopsy-attending & $6.7 \pm 2.0$ & $4.8 \pm 3.4$ & $0.01^{*}$ \\
\hline Number (proportion) of dry taps & $7.0(25.0 \%)$ & $4.0(15.4 \%)$ & 0.505 \\
\hline
\end{tabular}

*Indicates statistical significance

where an aortic sonogram and an echocardiogram were performed and reported normal. Two hours after the SBM, the patient was examined by a neurologist who found that the patient had completely recovered. The consultant felt unable to distinguish between an excess of local anesthetic adjacent to the right sciatic nerve versus an anxiety reaction to a difficult procedure. The patient was discharged from the emergency department and followed in clinic. She had no further right lower extremity weakness or numbness or other neurological symptoms. There were no other adverse events encountered in either study group.

\section{Discussion}

Since the introduction of the Jamshidi needle for BMPs 40 years ago, few technical advances have been made in the field. Commercial introduction of the OBM, a battery powered drill with attached needle for bone marrow aspirations and biopsies, followed FDA approval of the device in 2007. Preliminary studies indicated that the OBM was safe and yielded adequate aspirates in a short period of time. ${ }^{5}$ A prospective randomized study by Berenson et al., ${ }^{6}$ comparing OBM and SBM, indicated that the OBM yielded bone marrow biopsy specimens of significantly greater volume, in a shorter period of time, with less residual pain in adult patients. A prospective, randomized study of OBM versus SBM by Swords et al., ${ }^{7}$ using experienced operators, indicated that significantly longer biopsy cores were obtained with the OBM method. The mean biopsy lengths obtained in that study (13.1 mm OBM and $8.2 \mathrm{~mm} \mathrm{SBM)} \mathrm{were} \mathrm{very}$ similar to those obtained in the present study (15.3 $\mathrm{mm} \mathrm{OBM}$ and $9.8 \mathrm{~mm} \mathrm{SBM}$ ), as shown in Table 3.

Thus, the primary endpoint in the present study confirmed the observation that the length of the marrow biopsy is significantly longer with the OBM method than with the SBM method. The length of the marrow biopsy is widely regarded as a surrogate for biopsy quality. ${ }^{7}$ Since the only reason for subjecting patients to this painful procedure is to obtain diagnostic information, the device which yields the most must be regarded as superior. The fact that the primary endpoint of the study, the length of the marrow core, was objectively determined in the pathology laboratory (which had no involvement in the study and no information as to how individual specimens were obtained), underscores the objectivity and validity of the conclusion. An element of operator bias appeared unlikely, since neophyte hematologists are maximally motivated to obtain optimal marrow specimens. A preplanned interim analysis detected significant differences, and, as a result, the study was terminated early .

Other OBM studies have been conducted with experienced operators in clinical practice settings. ${ }^{5-7}$ The present study is the first to be conducted in teaching hospitals utilizing inexperienced operators, i.e. hematologists-intraining. The fact that the results were similar whether the operators were experienced or not confirms the ease with which the OBM technique is mastered. Indeed, the majority of the participating fellows expressed a preference for the OBM method which suggests that OBM 
is a better training tool for teaching programs.

The key secondary endpoint of the study was the duration of the procedure, skin-to-skin. The mean procedure time was significantly shorter with the OBM (174.6 s) than with the SBM (292.1 s). In other words, even with inexperienced operators, OBM changed BMP from a 5 minute procedure to a 3 minute procedure, on an average. Other comparative studies, employing experienced operators, have reported even faster OBM procedure times, and all have demonstrated significantly shorter times compared to a SBM control group. ${ }^{6-8}$ According to Kuball et al, BMP time is particularly relevant to patient pain. ${ }^{2}$ Patients are generally willing to undergo the BMP and a reasonable level of pain, providing that the procedure time is relatively short.

Pain scores, as reported by the patients, the fellows and the attending hematologists/ research technologist were also secondary endpoints of the study. The patient-reported pain scores showed a trend favoring $\mathrm{OBM}$, but the difference was not significant $(\mathrm{P}=0.11)$. A similar result was reported by Berenson et al who opined that the overall patient-reported pain score is largely dominated by the sharp pain of marrow aspiration. The latter would not be expected to differ between various needles. Both the fellows' and the attendings' perceptions of patient pain were significantly less with OBM compared to SBM, a result subject to observer bias. These data suggest there is a need for clinicians to reconsider current pain treatment options. These could include identifying patients at risk for significant pain during bone marrow procedures, discussing analgesia and even sedation options with patients including associated risks, and possibly re-dosing during the procedure, particularly if multiple punctures are required.

Interestingly, the OBM patients expressed a significantly greater willingness to have a repeat BMP than the SBM patients $(\mathrm{P}<0.03)$. That result may be criticized as possibly subject to physician influence. However, another possible explanation for the higher level of patient willingness to have a repeat procedure may be the difference in degree of procedure difficulty between the two procedure types. Kuball noted that technical difficulties showed a trend toward a more painful procedure. ${ }^{2}$ As shown in Table 3, the Fellows did have more difficulty with the SBM than with the OBM procedure.

Other secondary endpoints included the scores assessing ease/difficulty of the procedure by patients, fellows and attendings. On a scale of 0-10 for procedure difficulty, patients recorded a mean value of 1.1 for $\mathrm{OBM}$ and 2.2 for SBM. However, the difference was not statistically significant, owing to the wide standard deviation in both groups. On the other hand, the fellows and attendings rated the
OBM procedure as significantly less difficult than the SBM, a result subject to observer bias. The questionnaire scores regarding quality of marrow aspirates and biopsies from the fellows and the attendings/research technologist did not show notable differences between the OBM and the SBM groups and were also subject to observer bias.

Refusal by patients to undergo BMPs, especially in diseases like myeloma, leukemia and lymphoma, lead to delays in the diagnosis and treatment which may have fatal consequences. Any device, such as OBM, which promotes greater patient acceptance of a painful, but necessary, procedure may be anticipated to improve quality of care and to enhance favorable clinical outcomes.

Cost effectiveness is an ever present criterion in the field of medical devices. The data presented in this and other OBM studies leave little question as to the superior effectiveness of OBM when compared to SBM. In a study involving 767 patients, Bishop et al reported that only $42 \%$ of bone marrow biopsy specimens were long enough for definitive diagnosis. ${ }^{9}$ The following points are relevant to the cost portion of the equation. First, the battery powered driver has a negligible cost since it can be used for about 500 procedures. Second, the OBM sterile disposable trays retail for about $\$ 40$ more than many SBM trays. But the lower cost of SBM trays is offset by the inferior quality of the marrow specimens, delays in diagnosis and treatment, the necessity of repeating some BMPs, to say nothing of the longer procedure time and the inferior patient acceptance with the SBM. The long term costs and consequences of training future hematologists with inferior devices are not readily calculable.

\section{Limitations}

There were several limitations in this study. As with other device studies, this study was limited by the absence of blinding of patients , operators or observers. The noise and the vibration of the OBM driver limited ability to blind the study for the patients ; the operator or observer could not be blinded for obvious reasons. Although the primary endpoint (biopsy length) and a secondary endpoint (procedure time) were objectively determined, other secondary endpoints derived from the various questionnaires could not be free of observer bias or physician influence. There was also potential operator bias in the primary endpoint since bone penetration by the operator might be influenced by the fellow's personal preference for the SBM vs. OBM. Another limitation was including data from multiple marrows for different fellows, as opposed to one marrow of each type per fellow, which would have taken years to complete. There was also variability in the number of marrows performed by each fel- low, however this was partially offset by each fellow alternating between the SBM and OBM. Some senior fellows also had greater prior experience with the SBM before they were certified for the study. However greater experience with the SBM would have shifted the results in favor of the SBM rather than OBM. Finally, a detailed, blinded comparison of the pathologic quality of OBM versus SBM specimens was not carried out, owing to the omission of a specific consent phrase in the patient consent form. Nonetheless, blinded observations of the OBM and the SBM biopsy specimens by the hematopathologists did not reveal any notable differences in the amount of marginal necrosis (as might be caused by heat denaturation), hemorrhage, aspiration artifact, or crush artifact. Both OBM and SBM biopsy specimens displayed variable aspiration artifact, which was expected since the study design mandated a one puncture/one needle approach.

\section{Conclusions}

The results of this first prospective, randomized trial in two teaching hospitals comparing BMPs in OBM and SBM patients, as performed by novice hematologists, indicate that significantly longer and better quality marrow biopsy cores may be obtained in a much shorter period of time, and with less patient pain when using the OBM device. Adverse events were inconsequential. The slightly greater expense of OBM appears justifiable in balance. Wider acceptance of OBM as a preferred BMP device in teaching hospitals may lead to greater acceptance of BMP as a necessary procedure by patients, to improvements in the diagnosis and treatment of hematology/ oncology patients and better training for fellows.

\section{References}

1. Perkins SL. Examination of the blood and bone marrow. In Greer JP, Foerster J, Rodgers GM et al. Wintrobe's Clinical Hematology, Volume One. Philadelphia, PA; Lippincott Williams \& Wilkins, 2004:15-17.

2. Kuball J, Schuz J, Gamm H et al. Bone marrow punctures and pain. Acute Pain 2004;6:9-14.

3. Bain BJ. Morbidity associated with bone marrow aspiration and trephine biopsy-A review of UK data for 2004. Haematologica 2006;91:1293-4.

4. Alsop S. Stay of execution. Philadelphia, PA; Lippincott, 1973:19,70, 160.

5. Cohen SC, Gore JM. Evaluation of a pow- 
ered intraosseous device for bone marrow sampling. Anticanc Res 2008;28:3843-8.

6. Berenson JR, Yellin 0, Blumenstein B et al. Using a powered bone marrow biopsy system results in shorter procedures, causes less residual pain to adult patients, and yields larger specimens. Diagn Pathol 2011;6:1-6.
7. Swords RT, Anguita J, Higgins RA et al. A prospective randomised study of a rotary powered device (OnControl) for bone marrow aspiration and biopsy. J Clin Pathol 2011;64:809-13.

8. Miller LJ, Philbeck TE, Montez DF et al. Powered bone marrow biopsy procedures produce larger core specimens, with less pain, in less time than with standard manual devices. Hematology Reports 2011;3: 22-5.

9. Bishop PW, McNally K, Harris M Audit of bone marrow trephines. J Clin Pathol 1992;45:1105-8. 